UDC 681.3.06

D. I. Konotop ${ }^{1}$, desing engineer,

DOI: http://dx.doi.org/10.20535/0203-3771342017130222

V.P. Zinchenko ${ }^{2}$, candidate of technical sciences

\title{
3D-MODELS DESIGN CONCEPT OF COMPLEX TECHNICAL OBJECTS USING KNOWLEDGE-BASED TECHNOLOGY
}

Ua Створені за допомогою continuous acquisition and life cycle support (CALS, безперервна інформаційна підтримка життєвого циклу продукту) $3 D$-моделі складних технічних об'єктів (СТО) не повністю пов'язані між собою та для $3 D$-моделювання СТО використовуються різні компоненти $C A L S$, що приводить до проблем конвертації даних. Метою роботи $є$ удосконалення технології $C A L S$ для забезпечення зв'язку етапів побудови $3 D$-моделей $\mathrm{CTO}$ на основі знання-орієнтованих технологій протягом циклу їх створення та обміну даними між різними компонентами $C A L S$.

Ru Созданные с помощью continuous acquisition and life cycle support (CALS, непрерывная информационная поддержка жизненного цикла продукта) $3 D$-модели сложных технических объектов (СТО) не полностью связаны между собой и для $3 D$-моделирования СТО используются различные компоненты $C A L S$, которые приводят к проблемам конвертации данных. Целью работы является усовершенствование технологии $C A L S$ для обеспечения связи этапов построения $3 D$-моделей CTO на основе знание-ориентированных технологий на протяжении цикла их создания и обмена данными между различными компонентами $C A L S$.

\section{Introduction}

The development of complex technical objects (CTO) models is carried out by using information technology (IT), in particular technologies of continuous acquisition and life cycle support (CALS) [1], which is a complex of tools for their creation. Note that the models describe the relationship between parameters and characteristics of the CTO form and size.

CALS models of CTO have the following disadvantages. The models at different stages of the CTO creation are not fully interconnected; the different components of the CALS are used for CTO modeling, which leads to the problems of data conversion between the components and, in general, leads to partial or complete loss of model data, its history, topology and parameters [2].

In this case, there is a tendency to improve the CALS management by engineering knowledge (Managing Engineering Knowledge, MOKA) [3] based on Knowledge-Based Technologies (KBT) tools and methods. In particular, the analysis of CALS components showed that the developed knowledge bases

\footnotetext{
${ }^{1}$ ANTONOV Company

${ }^{2}$ Igor Sikorsky Kyiv Polytechnic Institute, Department of Experimental Research Automation
} 
(KB) are placed directly in CAx, which makes it impossible to use them in other CALS components. Therefore, an urgent problem is the need to develop a concept of a method for CTO constructing of models based on KBT for CALS, which will solve the above problems.

\section{Formulation of the problem}

The aim of the work is to improve the technology of CALS to provide communication between the stages of CTO models development based on KBT during their creation and exchange of data between different components of CALS.

\section{Stages of CTO modeling and its disadvantages}

The main components of CALS technology are computer-aided systems (CAx) and product lifecycle management (PLM) information systems (IS) that allow systematizing the storage and retrieval of data from CAx [1,2].

Existing CTO modeling process based on the integrated design and computer simulation method and on PLM parallel design technology is presented by the procedure:

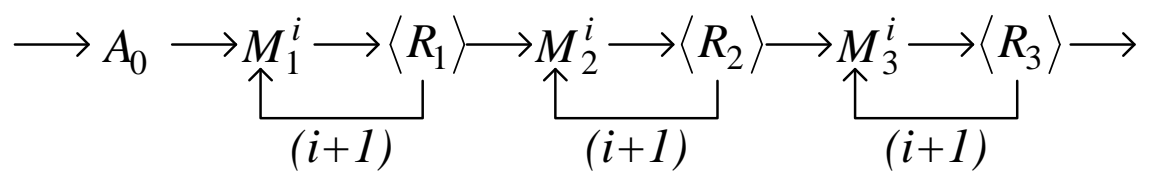

where $A_{0}$ - initial data (technical task, TT);

$M_{i}$ - model at the development stage: base control structure (BCS, $M_{1}$, including master-geometry model (MGM); objects allocation (OAM, $M_{2}$ ) and complete definition of CTO (CDM, $M_{3}$ );

$R_{i}$ - criteria for the development of each stage of the model development; $(i+1)$ - changes, refinements of each stage of the model development.

The analysis of the existing approach to CTO models constructing shows its ineffectiveness in that it is necessary to make changes continuously in the model, to change the data for the IS CAx and PLM [5]. It is necessary to add that:

- at each stage of CTO design, the developed model includes a large number of elements (in particular, at the stage of OAM development - up to 10,000) and the elemental parametric expressions, references, constraints, etc.;

- models of different stages of CTO design are not interconnected;

- models are related to other CTO models, but modifications in some models that have to bear the load on other models are significant and prone to errors.

- the interaction of IS technology CALS is complex, which is manifested in the problems of data converting from one component to another and leading to a partial / complete loss of history, topology and model parameters. 
Based on the data analysis, the following research objectives are set:

1. to perform the methods analysis for CTO models creating in CALS;

2. to develop a method of CTO models constructing based on KBT (hereinafter referred to as "knowledge-based modeling" ("KBM");

3 . based on the "KBM approach", to develop a parametric information management algorithm and an algorithm for data exchange between the components of CALS;

4. to develop tools and application software (AS) of "KBM approach";

5. to show the effectiveness of "KBM approach".

It is proposed to develop a "KBM approach" based on the ontological description of the process of CTO models creating taking into account the need to control the parametric information and data exchange between the IS CALS technology.

\section{Development of knowledge-based modeling approach}

In any CTO creating the constant control of the IT development is required. To do this, we developed a model for assessing the need for changes in existing IT. One of the methods based on peer review is SWOT analysis. An expert commission of 6 experts is created. To determine the strategy of further IT development of CTO models, each expert fills the matrix of the impacts of opportunities on the system benefits. To determine the strategy for further action of creating an effective IT design of CTO models, experts fill out a matrix of potential impact of strengths $(S)$ on the opportunities $(O)$. At the intersection of $S$ and $O$, an expert assessment of their mutual influence $\left(e_{i j}\right)$ is given in points from 0 to 5 (where " 0 " is "no influence", " 5 " - "the impact is very strong".) The total amount of points in the columns shows the priority taken into account or other factor when choosing a strategy: $E_{j}=\max _{j} \sum_{i=1}^{n_{S}} e_{i j}\left(j=\overline{1, n_{O}}\right)$, where $e_{i j}$ - element of the matrix to determine the optimal opportunity, $n_{O}$ - opportunities number, $n_{S}-$ strength number.

As a result, the ranking of opportunities takes the form: $O_{1}<O_{3}<O_{2}<O_{5}<\mathrm{O}_{4}$, indicating that the most important for the effective CTO creation is the revision of existing IT creation of CTO models for increasing competitiveness and the continuation of CTO models lifecycle. Based on the obtained expert assessment, an approach of knowledge-based CTO modeling is developed, that complements and extends the existing method of integrated simulation and computer design and parallel design of PLM technology to increase their efficiency in the process of CTO models creating.

A feature of the IT development task is the creation of a generalized model (GM) of CTO, which is used to optimize the process of models creating as components of CTO and CTO in general, that the number of criteria optimality $n>>1$ and the problem is solved by using methods of analysis, decomposition 
and synthesis. The problem of optimizing the process of creating CTO models is formulated as a task of the decision-making process, which involves three stages: "external" development (goals and objectives are defined, and requirements are created for its characteristics ensuring the achievement of these goals), "image formation" and "internal" development (realization of basic parameters of models).

PLM parallel design technology provides simultaneous access for all participants to the project implemented in PLM. The scheme of CTO project tree, supplemented within the method by the introduction of the component "Control models", is presented in fig. 1, where it is marked: KS - kinematic scheme, CS - construction scheme, SES - systems and equipment scheme, PP - power plant.

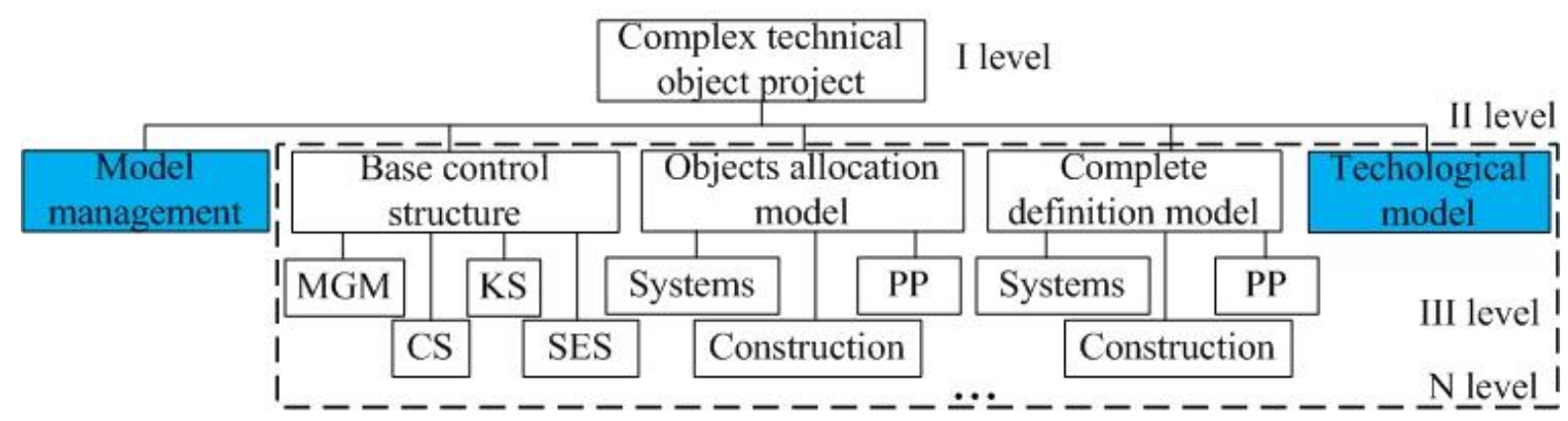

Fig. 1. Scheme of project tree of complex technical object in IS PLM

Let's denote $m_{G M}=\left(m_{1}, m_{2} \ldots m_{N}\right)$ - the set of GM components of CTO, each of which includes restrictions (geometric, technological, etc.), $m_{i} \in M_{i}$.

For a single criterion of efficiency $F_{i}\left(m_{G M}\right)$ models $m_{G M} \in M_{G M}$ the task of optimal process of CTO GM creating taking into account the restrictions imposed on the BCS stage, is to determine the parameters vector of the CTO creating process: $m_{G M}^{0} \in \operatorname{Arg} \max _{m_{G M} \in M_{G M}} F\left(m_{G M}\right)$, where:

$$
\begin{aligned}
& \operatorname{Arg} \max _{m_{G M} \in M_{G M}} F\left(m_{G M}\right)= \\
& \quad\left\{m_{G M} \in M_{G M} \mid F\left(m_{G M}\right)=\max _{m_{G M} ! \in M_{G M}} F\left(m_{G M}^{\prime}\right)\right\} .
\end{aligned}
$$

The development of the GM component of CTO is associated with great dimensionality of $N$ set of $m_{G M}$, which needs many resources to determine $F\left(m_{G M}\right)$. Therefore, (1) it is necessary to use the decomposition method for solving the task.

If $u_{i}\left(m_{G M}\right)(i=1,2, \ldots, e)$ are partial criteria for the quality of the process of CTO GM creating, $F\left(m_{G M}\right)$ monotonous (for $\forall m_{G M}, m_{G M}^{\prime} \in M_{G M}$ from $\left.u_{i}\left(m_{G M}^{\prime}\right) \geq u_{i}\left(m_{G M}^{\prime \prime}\right), i=1,2, \ldots, e \Rightarrow F\left(m_{G M}^{\prime}\right) \geq F\left(m_{G M}^{\prime \prime}\right)\right)$. Let's denote $\Pi(U)$ - set of optimal Pareto vectors from $U, \Pi\left(M_{G M}\right)$ - vectors set $m_{G M} \in M_{G M}$ for 
which $u\left(m_{G M}\right) \in \Pi(U)$, then the task of determining the vector of parameters of GM component of CTO takes the form:

$$
m_{G M}{ }^{0} \in \operatorname{Arg} \max _{m_{G M} \in \Pi\left(M_{G M}\right)} F\left(M_{G M}\right) .
$$

Consequently, the solution (1) can be represented as decomposition: the location of vectors $m_{G M} \in \Pi\left(M_{G M}\right)$ and the solution (2). In this case, the "image formation" stage does not depend on the criteria $F$, which allows the significant reducing of the variants number of GM of CTO at the stage of "external" development. As a result, at this stage, coordinated with the possibilities of "internal" development of CTO is formed.

The formalization of the existing CTO creation process was carried out and a generalized CTO model was developed through the maintenance of the knowledge base $(\mathrm{KB})$ and database (DB), and the use of the production model and its combination with CTO models through the maintenance of CTO project in the IS of PLM (fig. 2).

Fig. 3 shows a diagram of the activity of knowledge-based CTO modeling approach, which is the basis for the development of information technology, which complements and extends the existing method of integrated modeling and computer.

Data of the method are organized into structures: KB, containing the terms of CTO modeling, communication between them, rules that establish the order of interpretation of terms, the application of knowledge, the terms of conducting calculations, alignment with the requirements of standards. In addition, a file database containing parameters for determining the models of the SRT and DB of the enterprise containing CTO models in IS of PLM is presented.

\section{Knowledge-based modeling approach realization}

For implementation of the "KBM approach" the following tools have been developed: KB, DB, multiagent system (MAS), AS, IS of CALS have been selected, which allow the applied problems solving most effectively. A multiagent system, with the help of specially designed actions of software agents, can maintain duplex communication from the database at all times and enables to request and receive information about the rules, conditions and limitations of KB.

Ontology (O) of CTO models development can be represented as:

$$
O=<M_{G M}, R, F>\text {, }
$$

where $M_{G M}$ - set of concepts (terms) of the field of GM of CTO creation; 


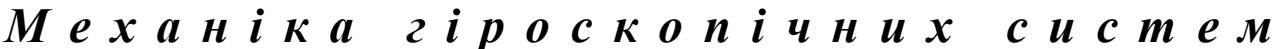

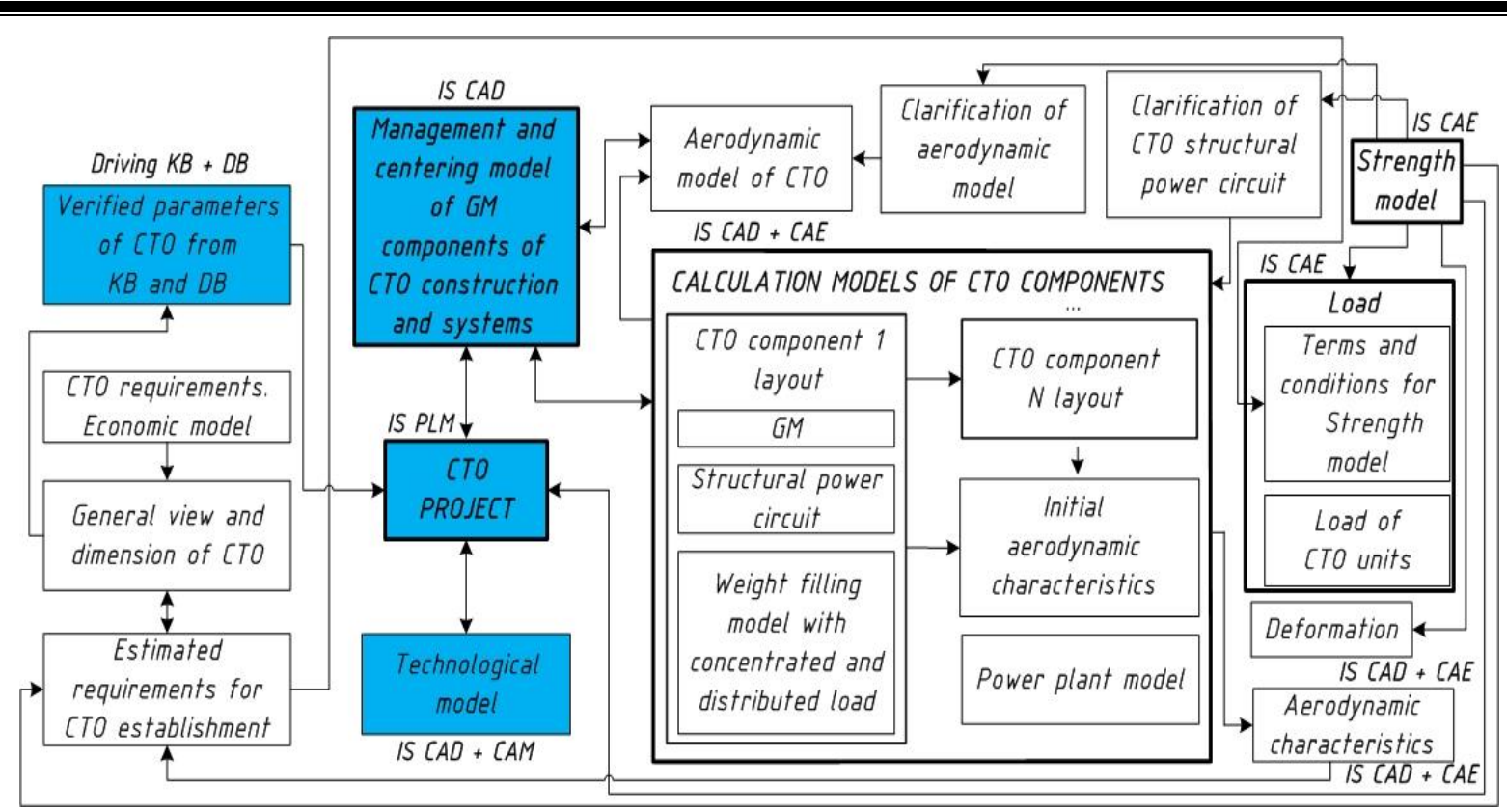

Fig. 2. A generalized model of complex technical objects

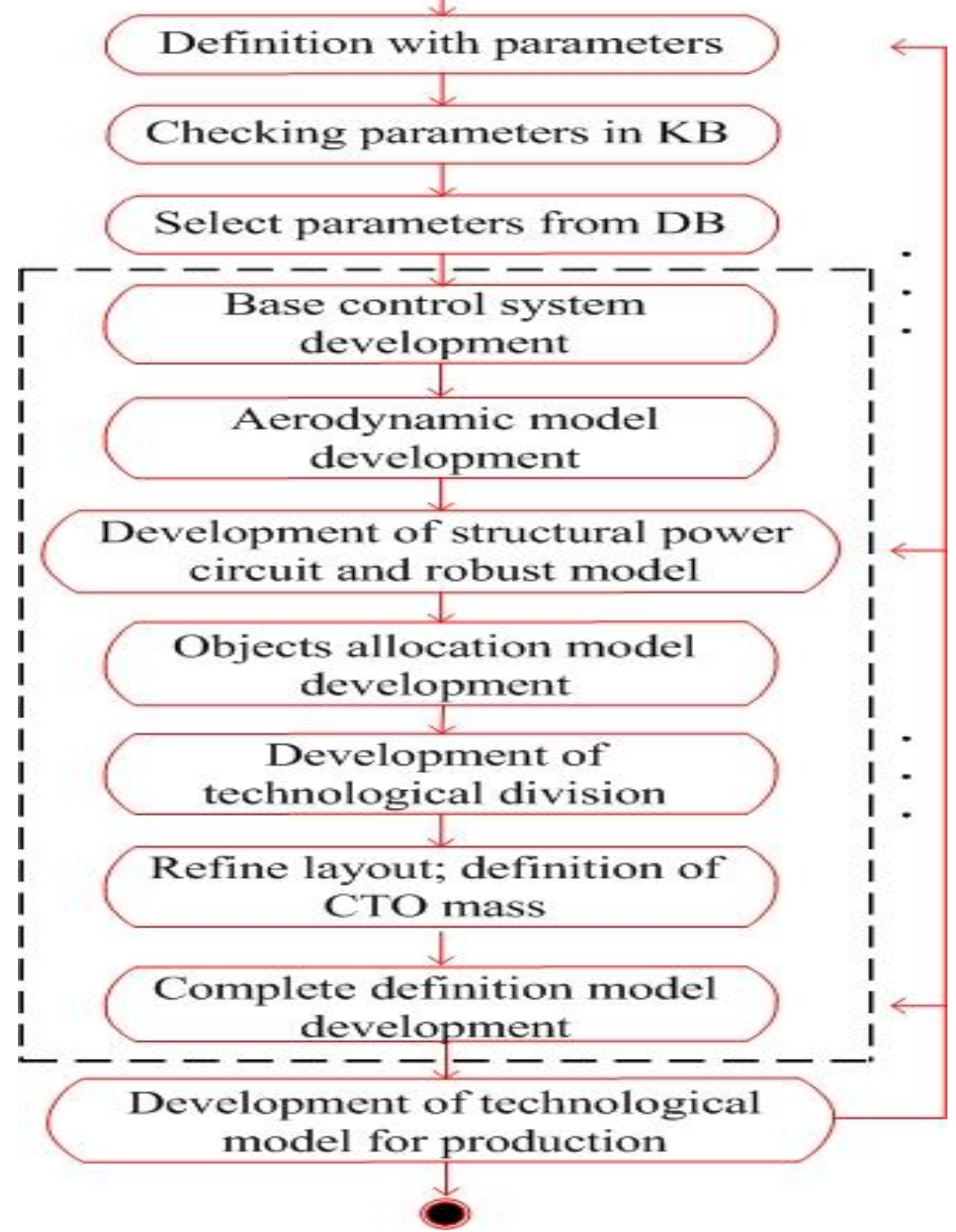

Fig. 3. Diagram of the approach's operation 
$R$ - a finite set of relations between concepts (terms) of the field of GM of CTO creation;

$F$ - complete set of interpretation functions, which are given on the concepts and attitudes of the ontology.

At the 0 -th level there is only one vertex that corresponds to the name of the domain "Development of CTO GM", any vertex of the following levels is associated with one vertex of the upper level and a definite subset of the vertices of the lower one.

In the developed $\mathrm{KB}$ the restrictions on components of the DB "Parameters of CTO models" are stored in accordance with the requirements of normative documents, TT, etc. The formulation of the purpose of KB and DB using is to determine the variants of solutions in the admissible set, which are described by constraints, which option is the criterion of optimality (target function or function) determining the quality of the decision, takes extreme value: $F^{*}=\underset{P_{n} \in E_{k}\left(m_{G M}\right)}{\operatorname{extr}} F\left(P_{n}\right)$, where $E_{k}\left(m_{G M}\right)$ - performance criteria $m_{G M} \in M_{G M}$. Each element of the model is described by its own set of parameters, and presented as a set of: $m_{i}=f\left(P_{i}\right)$, where $P_{i}$ - parameters of model: $P_{i}=\bigcup_{i=1}^{n} p_{i}$.

Overall, the system of restrictions looks like:

$$
\left\{\begin{array}{l}
E_{1}\left(m_{G M}\right) \geq 0 ; \\
E_{2}\left(m_{G M}\right) \geq 0 ; \\
\cdots \\
E_{k}\left(m_{G M}\right) \geq 0 .
\end{array} .\right.
$$

In particular, inequalities in the system $E_{1}\left(m_{G M}\right)$ are geometric; the specificity of CTO development also imposes restrictions: $E_{2}\left(m_{G M}\right)$ - centering; $E_{3}\left(m_{G M}\right)$ - CTO mass; $E_{4}\left(m_{G M}\right)$ - external influences on the area of development (levels of mechanical, climatic, etc. influences), compatibility of equipment; $E_{5}\left(m_{G M}\right)$ - fulfillment of technological requirements, which is a connecting link between design and production of CTO, etc. Acceptable levels $E_{i}\left(m_{G M}\right)$ stipulated by a number of normative documents, requirements for the establishment of CTO, TT, etc.

The developed rules in KB describe the connection between the models of CTO determined by the system of fuzzy output on the basis of fuzzy IF-THENELSE rules. Let's illustrate the example of the rules: IF $p_{i}^{\min }>p_{i}>p_{i}^{\max }$, THEN $p_{i}$ - INVALID (regarding restrictions of any models); IF $p_{G_{i}}$ CHANGE, THEN CHANGE $m_{G}, m_{W}, m_{A}, m_{C C}, m_{S}, m_{P P}, m_{T}, m_{E}$; etc.

Carrying out the computing environment survey and taking into account the world-wide experience of leading manufacturing companies, it is decided to use IS of PLM TeamCenter; The database is maintained in MySQL using the 
DbVisualizer database management system; Protege 4.3 and DbVisualizer are used to develop KB. In the work, the Java environment was created for the development of multi-agent systems and for connection of IS NX from the database and the Visual Basic environment for communication CAx CATIA from DB.

The IT architecture for generalized CTO model creation at the enterprise is shown in fig. 4 , where the joining of developed IT tools to the existing servers of the enterprise, from which the data is presented.

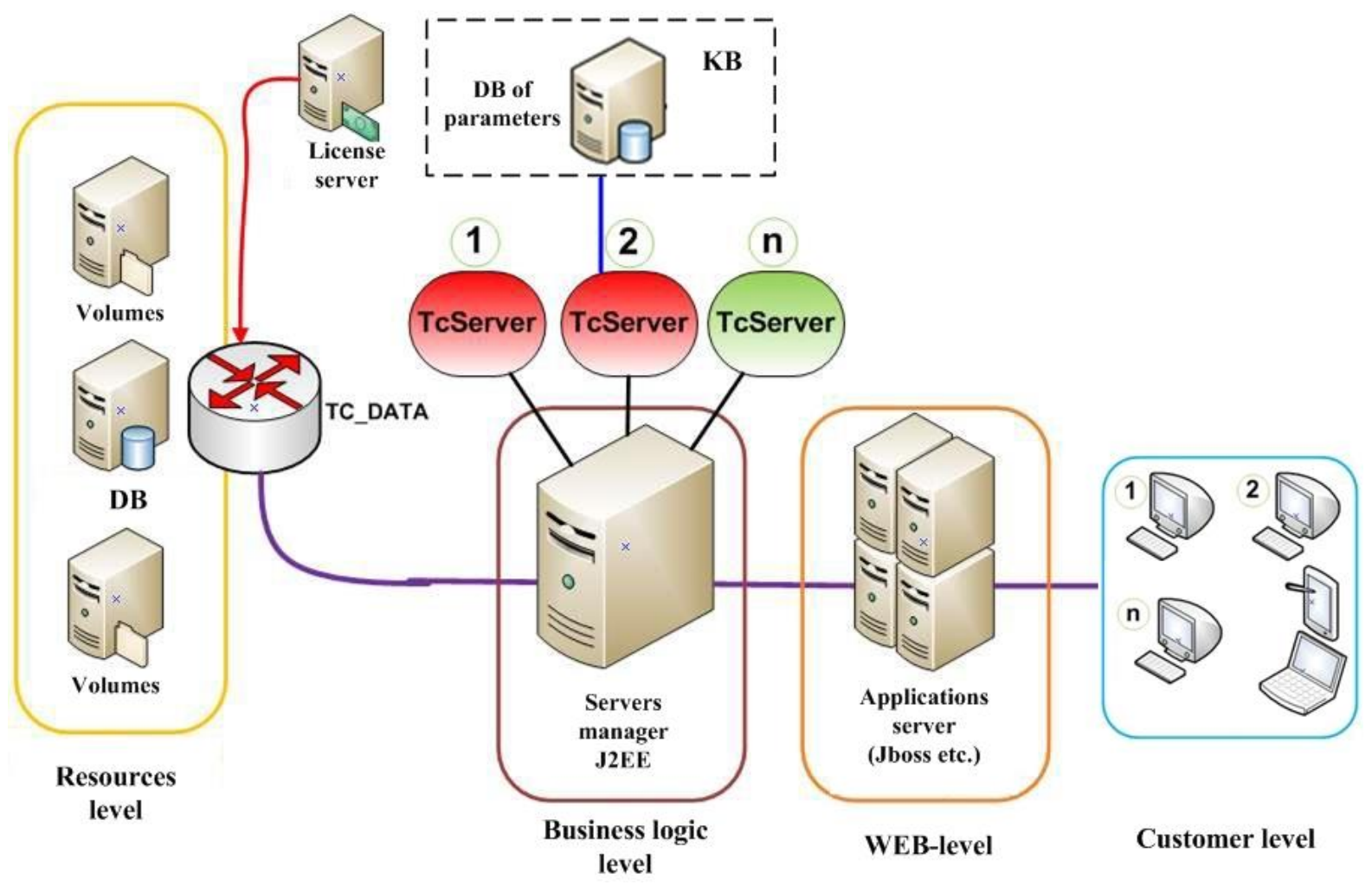

Fig. 4. Architecture of information technology for the creation of a generalized model of complex technical objects

Implementation of information technology for the creation of a generalized CTO model supplements parallel design technologies and CALS technology information systems for constructing models of complex technical objects, which provides a shorter term for the CTO creation by managing the parameters of CTO model, which provides approximately $22 \%$ savings in material costs. In addition, this is only considering the aspect of applying the algorithm of the method for managing parametric information. If the company uses other IS CAx, the proposed algorithm for the method for data exchange between different IS of CALS will provide additional efficiency of using the method due to the fact that there is only one KB and DB - common to all used IS of CAx and PLM. 


\section{Conclusions}

The actual scientific and technical problem of CALS technology improving is solved to ensure the communication between the stages of CTO models development based on KBT ("KBM approach") during the cycle of their creation and data exchange between different components of CALS.

The results of the study suggest the following conclusions:

1. The review and analysis of the methods of CTO models creation in CALS during the life cycle of CTO are carried out.

2. The concept of the CTO models development method based on KBT ("KBM approach") is developed.

3. Based on the "KBM approach" algorithm for managing parametric information and algorithm for data exchange between components of CALS are developed.

4. The "KBM approach" and application software are implemented.

5. The efficiency of the "KBM approach" using is shown on the example of the aircraft modeling.

\section{References}

1. Information support for science-intensive products. CALS-technologies / I. P. Norenkov, P. K. Kuzmyk - M.: Izv-MGTU named N. E. Bauman, 2002. $-319 \mathrm{pp}$.

2. Information technology in high-tech engineering: Computer support for industrial business / Under the general. Ed. AG Bratukhin - K.: Technics, 2001. $-728 \mathrm{p}$.

3. Stokes M. Managing Engineering Knowledge MOKA: Methodology for Knowledge Based Engineering Application, Professional Engineering Publishing, 2001. $-298 \mathrm{p}$.

4. Zinchenko V. P. Information technologies for modeling the layout of a complex technical object / V. P. Zinchenko, D. I. Konotop, A. P. Sidorenko, V. V. Borisov // Information systems, mechanics and control. - 2011. - № 6. - P. 27-35.

5. Zelensky V. A. Designing of complex systems. - Samara: Samar. state aerospace Un-t named S. P. Korolev (national research university), 2012-96 p.

6. CAD/CAM/CAE Observer № 1 (85) / 2014, Riga, Latvia, P. 30-36.

7. Mastering oracle scheduler in Oracle 11g Databases. Ronald Rood, 2009. $240 \mathrm{p}$.

8. Gruber T. R. (1993). A translation approach to portable ontologies. // Knowledge Acquisition. № 5(2). P. 199-220.

9. Stoyan Yu. G. Mathematical models and optimization methods for geometric designing / Yu. G. Stoyan, S. V. Yakovlev. - Kyiv: Scientific Opinion, 1986. -259 p. 